\title{
Exploring the Needs and Ways to Use Virtual Reality to Understand Animals' Perceptions
}

\author{
A Field Study in a Science Workshop and Exhibition
}

\author{
Haruka Kasuga \\ CoSTEP \\ Hokkaido University \\ Sapporo, Hokkaido, Japan \\ felisfelis@eis.hokudai.ac.jp \\ Haruna Kitamura \\ CoSTEP \\ Hokkaido University \\ Sapporo, Hokkaido, Japan \\ haru_neko@icloud.com
}

\author{
Machiko Ohashi \\ CoSTEP \\ Hokkaido University \\ Sapporo, Hokkaido, Japan \\ makomachikodayo@gmail.com
}

\author{
Masataka Yamamoto \\ CoSTEP \\ Hokkaido University \\ Sapporo, Hokkaido, Japan \\ ma28sa11@frontier.hokudai.ac.jp
}

\author{
Yusuke Konishi \\ CoSTEP \\ Hokkaido University \\ Sapporo, Hokkaido, Japan \\ shachi11ichigo@gmail.com
}

\author{
Takashi Murai \\ CoSTEP \\ Hokkaido University \\ Sapporo, Hokkaido, Japan \\ murai@costep.hucc.hokudai.ac.jp
}

Graduate School of Information

Science and Technology

Hokkaido University

Sapporo, Hokkaido, Japan

nadametic@gmail.com

\begin{abstract}
Knowledge of how animals perceive the world can provide a method of understanding them. Recently, it has become easier to experience augmented vision, particularly using head-mounted display virtual reality (HMD-VR). Research on the use of HMDVRs for applications in education has been increasing. However, it is impossible to express other animals' vision to humans with complete accuracy. To explore the needs and the appropriate methods of utilizing HMD-VR for learning animal vision, we created VR videos of eyesight, color vision, and dynamic vision filmed in a real environment and conducted a field study with the videos in a workshop at a zoo and an exhibition in a science festival. A questionnaire answered by 39 participants at the workshop and 196 attendees at the exhibition revealed that the animals and information sought by participants differed by participant demographics.
\end{abstract}

\section{CCS CONCEPTS}

- Social and professional topics $\rightarrow$ User characteristics; - Human-centered computing $\rightarrow$ Haptic devices; Field studies.

\section{KEYWORDS}

field study; head-mounted display (HMD); virtual reality (VR) in education; animal vision; animal-computer interaction (ACI); science communication

\section{INTRODUCTION}

Every living organism perceives the world in its own manner [1]. However, humans often forget this difference, resulting in

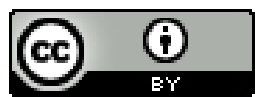

This work is licensed under a Creative Commons Attribution International 4.0 License.

ACl'2020, November 10-12, 2020, Milton Keynes, United Kingdom (C) 2020 Copyright is held by the owner/author(s).

ACM ISBN 978-1-4503-7574-0/20/11.

https://doi.org/10.1145/3446002.3446120 anthropomorphism, which can cause problematic misunderstanding of animal needs [2]. Animal-computer interaction $(\mathrm{ACI})$, which has been spreading rapidly worldwide since 2011 [3], involves design for use by animals [4, 5, 6, 7] and the application of technology to deepen human understanding of animals [8].

Because of the rapid advances in technology recently, a growing number of tools have become available that can help humans better understand animals. Fass and Walker suggested the importance of ACI applications in understanding how animals behave during their interactions with humans by designing content that allows humans to experience the sensations felt by animals, using pigeons as an example [9]. Examples of these content and applications include pictures taken using a bird-like eye from a drone or experiencing an immersive world of images using a head-mounted display (HMD). Moreover, the number of studies on applying HMDs for educational purposes across various fields has been exponentially increasing [10] because the immersive power of HMD-virtual realities (VRs) enables people to comprehend information that may be difficult to explain verbally. Therefore, we aimed to improve educational efficacy of HMD-VR by depicting how animal vision can be reproduced in VR. Providing opportunities to learn animal vision leads to an understanding of the animal and learning the appropriate design for that animal. This is an important application of ACI as it relates to animal welfare. However, humans cannot completely perceive animal vision because of the following factors: 1) limitations of human perception, 2) lack of technology for visual reproduction, 3) lack of research on animal vision, and 4) lack of understanding of all components of vision, such as visual acuity and visual field. Using HMD-VRs as tools to study animal vision is associated with the risk that the tools can cause new misunderstandings and biases. To introduce HMD-VRs to animal vision, appropriate instruction is required. However, suitable instruction will differ depending on the demography of people using HMD-VRs.

To explore the demands and appropriate methods of using HDMVRs as tools to promote awareness of the differences between humans and animals, we made three videos that can be viewed with an HMD and conducted a field study at two different science 
events. Then, we explored whether the contents must be varied depending on various demographics.

\section{BACKGROUND}

Creating scientific events that emphasize environmental issues and appropriate husbandry for animals as subjects is one approach to "foster promoting understanding between humans and animals" [3]. We conducted a field study at two science events of different natures: NoMaps and Equimonia.

\subsection{NoMaps}

NoMaps ${ }^{1}$ is a convention that highlights creative ideas and technology. It is held in Sapporo, Japan, which was designated as a "Creative City of Media Arts" by the UNESCO Creative Cities Network (UCCN) ${ }^{2}$. The convention is a week-long festival that has been held every October since 2016 and features a film festival and technology exhibition. Since 2017, NoMaps has cooperated with universities to hold VR-based science events. It held a science workshop for children at the Sapporo Science Center $^{3}$ in 2017 to allow children experience various organisms and their habitats through VR videos filmed by scuba divers in the waters of Lake Shikotsu. Finally, the children could take a stickerstyle quiz regarding the habitat of animals. This is an example of the application of VR to natural science education. The workshop won the Asahi VR Award ${ }^{4}$ hosted by The Asahi Shimbun Company, one of the largest journalistic organizations in Japan.

\subsection{Equimonia}

Equimonia ${ }^{5}$ is a festival held every November or December for enthusiasts of living organisms, including independent researchers. It includes three parts: "Buying and Selling," where artists and enthusiasts of living things buy and sell their works; "Viewing," where they exhibit their works; and "Knowing," where researchers give invited talks (e.g., "Looking for an antshaped beetle in the ant's nest" by Professor Maruyama from the Kyushu University Museum, and "A plant that has given up photosynthesis for a 'cunning' NEET life" by Professor Suetsugu from Kobe University). At each booth, exhibitors sell or display accessories such as creature motifs, laboratory equipment, selfpublished books, and specimens. The processes of buying, selling, and displaying works is similar to that of self-published articles and the accessory sales booth areas of the Comic Market ${ }^{6}$, the world's largest exhibition and sales event of fan products.

Because Equimonia's committee focuses on both popular companion animals and various living species, including unicellular organisms and fungi, the committee uses a $9+2$ axoneme that exists in the cilia of typical organisms as a motif for their $\operatorname{logo}{ }^{7}$. Equimonia's popularity is proven by the fact that thousands of fans, some of whom love "peculiar" living things, visit Equimonia yearly.

\footnotetext{
${ }^{1}$ https://no-maps.jp/

${ }^{2}$ https://en.unesco.org/creative-cities/

${ }^{3}$ http://www.ssc.slp.or.jp/

${ }^{4}$ http://www.asahi.com/shimbun/medialab/vraward/

${ }^{5}$ https://www.equimonia.net/

${ }^{6}$ https://www.comiket.co.jp/info-a/TAFO/C98TAFO/cmkfor.html

7 https://www.equimonia.net/2018/04/24/21/
}

\section{RELATED WORKS}

\subsection{Encouraging Empathy with New Technology}

To understand others' perceptions, it is important to actually experience these perceptions. Under the guidelines issued by the World Health Organization (WHO) ${ }^{8}$, various activities have been undertaken to encourage public understanding of people with disabilities, including their experiences; numerous studies have shown that educational activities that showcase the experiences of people with disabilities are effective [11].

For example, Väyrynen et al. developed a VR system that allows users to experience various visual impairments [12]. This helps us understand the challenges faced by each visually impaired individual. These VR experiences can create opportunities for humans to rethink how social environments are designed.

Oki et al. developed a device that allows the wearer to experience the perceptions and sensations of a smaller person and conducted both laboratory research and field research in nursing schools and museums to examine how the device modifies the wearer's body expression and experience [13]. The subjects wore an HMD and used a camera attached near their waist to perceive and explore the real world as a smaller person. The subjects in this study performed unusual behaviors and interactions. There were examples of childlike behavior among the wearers, suggesting that they were immersed in children's senses. Therefore, it is believed that such devices can help designers and teachers understand human beings with different perceptions of real-world environments.

Recently, applications have been developed to promote the understanding of both humans and animals. Webber et al. implemented and exhibited a Kinect-based drawing app for orangutans at a zoo [8]. Analysis of observations and interviews indicated that the visitors responses were cognitive and affective and the visitors exhibited motor empathy toward the animals, suggesting that the design was effective in two ways: 1) utilizing the animals' cognitive abilities and 2) reflecting individual preferences. One reason why this type of empathic reaction was triggered is that the drawing app prompted consideration of animals' cognitive processes and emotional states. Some visitors were surprised, and their perception of the orangutans' intelligence was renewed. Thus, providing awareness through new technologies will likely generate further interest in visitors.

In summary, new technologies seem to be useful in promoting an understanding of other organisms, regardless of species. These technologies will lead to important applications of ACI that will facilitate human-animal relationships.

\subsection{VR Exhibition in a Museum}

VR has been employed in multidisciplinary areas, such as education, culture, and arts and has been explored for educational use in museums, cultural centers, and entertainment facilities [14]. Particularly in museums, VR is considered useful as it allows visitors to move through time and space while remaining in the museum.

In terms of tourism, VR is also useful as a conservation tool and

8 http://www.who.int/disabilities/world_report/2011/en/ 
Exploring the Needs and Ways to Use Virtual Reality to Understand Animals' Perceptions

has been discussed as a potential substitute for actual visits to threatened locations [15]. The authenticity of a VR experience has also been a topic of much discussion; although VR essentially has no authenticity, the level of authenticity is subjectively determined by a user, and the experience is considered authentic depending on the context and direction.

Recently, the TNM and TOPPAN Museum Theater ${ }^{9}$ installed a permanent VR cinema that allows visitors to enjoy parts of the exhibits that are not normally viewable. Thus, VR has been effectively implemented in modern museums.

The use of VR in museums provides a new experience for visitors and promote an understanding of the content and a break from stereotypes.

\subsection{Education via VR}

The use of VR in the field of education has also garnered increasing interest, with an increasing number of papers and studies focusing on VR for educational purposes [10].

The prerequisites and issues for the use of HMD-VR in $\mathrm{K}-12$ schools have been explored from a teacher's perspective [16]. Although HMD-VR can make teaching and learning more interesting and effective, it is also more expensive and requires more staff and teachers' own knowledge of HMD-VR.

In some studies, VR has been introduced into educational settings, and its effectiveness was confirmed. Jackson and Fagan introduced VR-based learning content to 56 ninth-grade students and divided them into three groups: (1) studying alone with minimal information, (2) paired with other participants, and (3) paired with an expert [17]. The results showed that they could learn well despite a deficit in the knowledge of VR usage.

In addition, the usefulness and educational effects of VR in STEM education have been discussed [18].

In physics learning, it has been reported that gamified simulations on VR allowed students to repeat experiments without the risk of damaging the equipment while increasing learning motivation [19]. However, VR is not considered a substitute for a teacher; it can only complement the teaching process.

VR simulations to teach how to use laboratory instruments have been shown to be easy to navigate, to be as convenient as a physical laboratory, and to be comfortable because environments can be navigated without the need for additional support from investigators [20].

Blazauskas et al. reported that when they had 16-17-year-old students explore historical areas and answer questions regarding history in VR, the rate of correct answers to questions was higher than that for students who learned in a traditional classroom [21]. In addition, they confirmed that it promoted the motivation of students to learn about history by giving them opinions such as "I want to visit the historical area."

Fabola and Miller showed VR recreation of the fourteenth-century St Andrews Cathedral (Scotland) to 30 students in the first year at secondary school and then administered a questionnaire [22]. The results showed that VR increased the students' interest to learn history and that VR was easy to use regardless of experience, confirming its usefulness. The authors also compared HMD-based VR with VR on a screen, highlighting that there was no

${ }^{9}$ http://www.toppan-vr.jp/mt/en/about/

\section{ACI2020, November 2020, Milton Keynes,} United Kingdom

significant difference in the sense of immersion between HMDbased VR and VR on a screen that allow users to fly around freely. HMD-based VR allows people to explore ruins only from a pre-defined perspective due to the device's performance; whereas, mobile VR devices have the advantage of being able to compare recreated fourteenth-century ruins with the real heritage site in the field. Thus, it is meaningful to compare VR in an environment where real animals and exhibits are nearby, such as in a zoo or museum.

A VR gorilla exhibit was hosted at the Atlanta Zoo to provide hands-on educational tools for middle-school students to learn about gorillas' interactions, vocalizations, social structures, and habitats $[23,24]$. Users could pretend to be adolescent gorillas and live as members of a gorilla family while learning about gorillas' ecology through multimedia information. The results confirmed the educational effectiveness of this virtual environment.

These applications make good use of the immersive power of VR. By contrast, numerous previous studies have attempted to eliminate misunderstandings by providing additional information about learning in VR. It was also pointed out that VR only has a complementary role to teaching. Therefore, content-based instruction can be considered important.

\section{PRODUCTS}

Under researcher supervision, we produced the following three VR videos as an expression of art:

- Eyesight of turtles and tortoises

- Color vision of cats and dogs

- Dynamic vision of frogs and geckos

The elements of animal vision are diverse. Among them, we adopted "visual acuity" and "color vision," which makes it easy to understand intuitively the differences between animals, and "dynamic vision," which can be reproduced by video editing without special equipment. Although these videos do not represent the actual vision of animals with $100 \%$ accuracy, and the references regarding animal vision may not be entirely correct, utilizing the immersive power of watching VR videos can provide opportunities to imagine and consider the structure of the eye and the process of evolution by surprising participants with a glimpse of the differences in vision between humans and other animals. Therefore, the topics on which the VR videos were developed were decided based on whether we could express the differences in vision between humans and animals to at least the minimum required extent with our editing technique.

We used GoPro Fusion, a $360^{\circ}$ digital camera, to record each video with permission from each animal's owner. The equipment and videos were used to passively observe the animals. Considering the appropriate length for workshops and exhibitions, the three videos were made of the same length (approximately 2.5 min). The videos were edited to show tickers in three places to guide the direction in which the animals appeared, to provide explanations, and to prompt questions regarding the animals' ecology and vision. In our workshops or exhibitions, we played our VR videos on a smartphone (ZTE Blade V580 [Android 5.1; $77.2 \times 8.55 \times 155.3 \mathrm{~mm} ; 165 \mathrm{~g}$; RAM $2 \mathrm{~GB}$; ROM $16 \mathrm{~GB}$; $1080 \times 1920$ pixels full HD]) set in a VR headset (TEPOINN 3D VR headset [12 $\times 17.5 \times 24 \mathrm{~cm} ; 594 \mathrm{~g}]$ ) for a smartphone, and the 


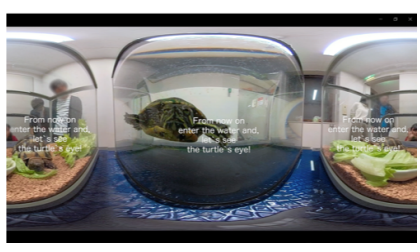

Figure 1: Beginning of our VR video on turtles' and tortoises' eyesight.
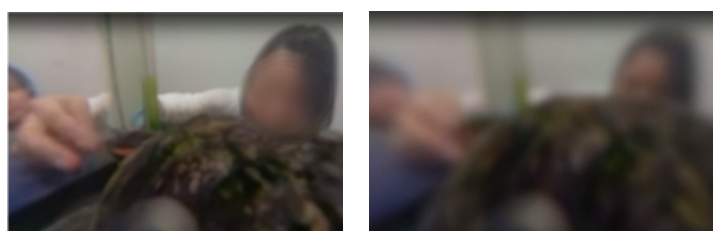

Figure 2: Before shading (left) and after the process (right).

smartphone application used was VR Player (Free).

\subsection{Eyesight}

We focused on the ability to sense the spatial frequency of the eyesight. The eyesight of an animal can be measured using a black and white striped pattern. As the number (density) of stripes increases, the black and white stripes are averaged, eventually making the stripes invisible as if the pattern were gray. Eyesight can be defined by the limit at which these stripes can still be seen; the number of stripes per visual angle is called the spatial frequency [25].

Eyesight (spatial frequency) varies between species and reflects ecological differences [26]. For example, predatory birds tend to have good eyesight in proportion to their height to find prey such as rats on the ground while they are flying high in the sky [27]. Meanwhile, bats live in the dark and have poor eyesight. Instead of relying on eyesight, bats are known to "see" the world using ultrasonic sound.

The eyesight VR video focuses on the chelonian. The eyesight of a turtle was measured using a physiological method called visual evoked potential (VEP) [28].

Turtles and tortoises are popular companion animals. The purpose of the video was to help people consider how different habitats of chelonians could affect their eyesight.

For the expression of this movie, we applied a blurring process using a Gaussian function of Adobe Premiere Pro Creative Cloud 2018 and asked three people with visual acuities of $2 / 20-4 / 20$, which is almost the same as that of a turtle, to judge the extent of blurring with and without their glasses. It is inevitable that there are limitations regarding the accurate expression of animal vision. For example, this process could not express the phenomenon of myopia, which is when a subject can see nearby objects clearly whereas distant objects appear blurry.

The camera starts between tortoises (Eurotestudo hermanni) and a turtle (Trachemys scripta), as shown in Fig. 1. Next, we go to a turtle aquarium and watch the humans outside from inside the aquarium. Then, the teleprompters explain that the eyesight of this species of turtle is worse than that of humans in general; the environment then becomes blurred, as shown in Fig. 2. Next, the environment shifts to the tortoise aquarium. Finally, teleprompters ask a question that encourages video watchers to imagine and guess how the difference in habitat could affect the eyesight of

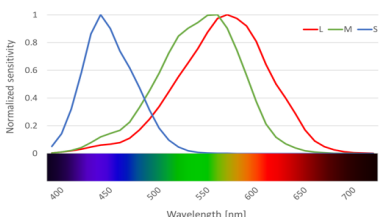

Figure 3: Wavelength representing the characteristics of trichromacy in humans.

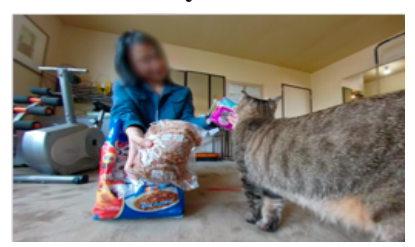

Figure 5: The photo on the left depicts trichromacy and that on the right depicts the color vision of cats and dogs.

chelonians.

Although there is no actual answer to the question "which chelonian has better eyesight?", as far as we know, our aim was to trigger the imagination of video watchers. We suppose that there are various ways to understand this: "the eyesight of tortoises could be better because it is easier to see when on land" and "aquatic turtles often come on shore; thus, the vision of the two different chelonian species hardly differs."

\subsection{Color Vision}

Color vision is the ability to detect and analyze changes in the wavelength composition of light [29]. Although color vision is affected by both physical receptors and the context in which an object is observed, which can cause optical illusions, in this research, we simply focused on the set of different types of cone cells. Light of different wavelengths is perceived as different colors when absorbed by cone cells in the eye. Humans have three types of cones that are most sensitive to light of short, medium, and long wavelengths, called S, M, and L cones, respectively [29]. They are also called blue, green, and red cones, respectively, as Fig. 3 shows; having different sets of cones means seeing the world in various colors.

In the video, we focused on cats and dogs because they are two of the most popular companion animals, and previous studies have focused on their color vision $[30,31]$. Most people have the three types of color cones shown in Fig. 3. Meanwhile, cats and dogs only have the $\mathrm{M}$ cone, which means that they see the world as a minority of people with deuteranopia color vision do, as shown in Fig. 4. For primates, trichromacy seems to have advantages when looking for fruits. For example, distinguishing an apple or orange from green leaves is easier with trichromacy. As this example implies, the distinctiveness of colors is believed to have evolved based on how each creature lives.

Understanding which color combinations are easily distinguishable is important to understand further cats and dogs that are often considered members of a family or working animals, such as guide dogs. In our introductory explanations to the viewers, we attempted to make them imagine the "universal design of color for the animals that live with us" by giving examples of the color-coding of dog-agility equipment and a touchscreen device for working dogs [32]. 
(a) Original frame

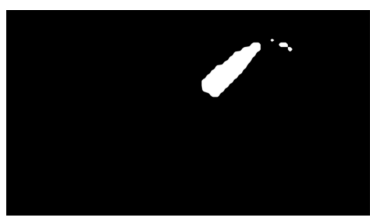

(c) After morphology processing
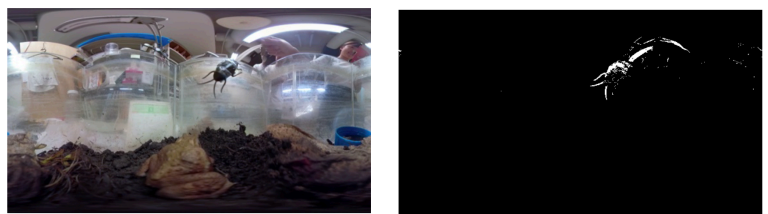

(b) Difference image generated with MOG 2

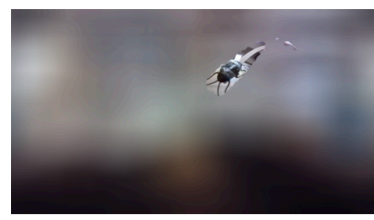

(d) Blurring of non-moving parts

Figure 6: Processing process for a frog's vision in VR videos.
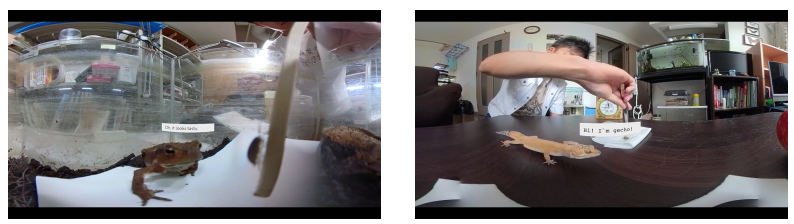

Figure 7: The feeding scene featuring the gecko and frog.

To express color vision with a lack of $\mathrm{M}$ cone cells in our video, we applied the transform matrices proposed in a previous study [33] to express deuteranopia.

The video begins with a trichromatic scene of a cat playing with catnip indoors and then a scene of feeding time when the cat eats its food. The same scene is then replayed in a deuteranopic style. The next scene is a trichromatic segment of a dog playing in a park. Then, the same scene is replayed in deuteranopic color vision. We presented the same scene in two different color visions to encourage viewers to understand the colors cats and dogs can distinguish, as shown in Fig. 5.

\subsection{Dynamic Vision}

Dynamic vision is the general term for the ability to see moving things. Here, we focused on very simple dynamic vision, the critical flicker-fusion frequency (CFF), and interesting features of the dynamic vision of frogs. CFF is examined by measuring the number of times one can identify a flashing of light in one second. CFF has been researched in many animal species; for example, the CFF for dogs is $80 \mathrm{~Hz}$, that for geckos is $20 \mathrm{~Hz}$, that for leatherback sea turtles is $15 \mathrm{~Hz}$, and that for squirrels and pigeons is $>100 \mathrm{~Hz}$ [34]. Therefore, dogs can see LED traffic lights, which generally flicker at approximately 100 times per second, as blinking, whereas people can only see the light as glowing. Thus, differences in the CFF between animals can alter their perceptions of the world.

Frogs are often well-known to have dynamic vision. The retinas of frogs have photoreceptors that can detect brightness and movement, but much of the visual information is removed from the outputs from the retina to the optic nerve. There are only four outputs to the optic nerve: standing edges, curvatures, changing contrasts, and local lessening of light intensity [35]. Therefore, it is believed that frogs can rapidly respond to moving objects but are less likely to recognize objects that do not move.

We did not have any method to let humans perceive a higher CFF world than our own, as we could not surpass human limitations. Therefore, we focused on the CFF of geckos, which is $20 \mathrm{~Hz}$. To express the world at a CFF of $20 \mathrm{~Hz}$, we reduced the frames per second (FPS) of the video using Adobe Premiere Pro Creative Cloud 2018. However, even at one-third of the human CFF, it would be difficult for humans to perceive the difference based on stroboscopic movement, the phenomenon in which people see videos as a flick book with 24 FPS continuous movement. To help the video viewers note the difference in the CFF, we put a clock with a second hand in view.

We created a VR video based on a frog's vision. In this video, we used a motion detection algorithm based on a method called MOG $2[36,37]$ with OpenCV in Python to completely blur non-moving objects. MOG 2 is a type of background subtraction used for detecting moving objects by sequentially constructing a background model from past frames using a mixed normal distribution and taking the difference from the current frame. By creating sequential background models, areas without moving objects can be prevented from being detected as differences due to illumination changes and shadow reflections. In other words, detecting a moving part (bug toy in Fig. 6) from a frame in a moving picture, as shown in Fig. 6 (a), only requires knowing the part that is not moving for a specific number of previous frames. As a result, Fig. 6 (b) shows a difference image in which the portions assumed to be moving are represented in white. Because the difference image contains distortions due to detailed changes such as lighting, noise must be removed by openly processing morphological transformations. Additionally, in the difference generated with MOG 2, only the outline of the moving object is detected; hence, it is necessary to fill in the undetected parts inside the contour using a closing process for morphological transformations. Therefore, as shown in Fig. 6 (c), the moving area can be extracted from the moving image and only the unmovable area can be completely blurred, as shown in the eyesight section of this book. Then, it becomes possible to express the frog's vision, as shown in Fig. 6 (d).

The story in the video is as follows: To evoke their movement, we took the video during feeding time, as shown in Fig. 7. The camera starts with a scene on the table where the geckos appear at $60 \mathrm{~Hz}$. Then, after the teleprompters appear, the FPS is turned down to $20 \mathrm{~Hz}$. Then, to explain the meaning of CFF, FPS is reduced to $5 \mathrm{~Hz}$. Next, the scene is changed to one of a frog eating bugs, recorded using a camera inside the aquarium under clear vision. Then, the scene shows the dynamic vision of a frog.

Our aim in this video was to increase the interest of the viewers; for example, we wanted to explain CFF by asking the question "Does the sense of the speed of time vary with your eyes?" and to explain the frog by asking "Which animal has a greater advantage: those seeing only moving objects or those also seeing stable objects?"

\section{FIELD STUDY}

We utilized our products for two events with different characteristics. One was a workshop as a part of NoMaps 2018. This was held for elementary- and middle-school students at a zoo. The second was a booth exhibition in Equimonia 2018. In both fields, participants could observe various "real" animals before and after their experience of our VR products. A synergistic effect due to observation of real animals combined 

Kingdom

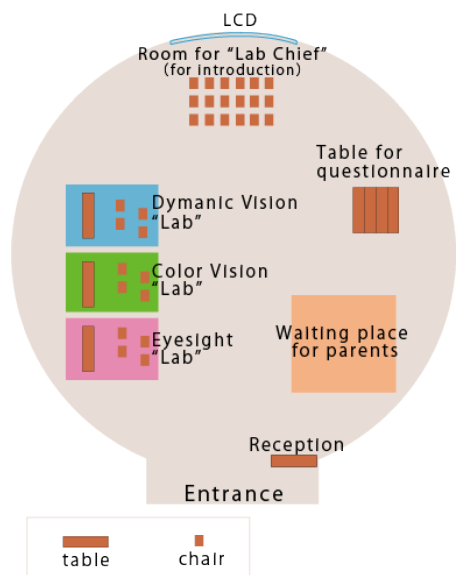

Figure 8: Apparatus in each corner of the workshop.

with the experience of the virtual animal vision was expected based on suggestions from previous studies [21,22].

\subsection{Zoo Workshop in Cooperation with NoMaps}

The workshop was held in the zoo information hall (Fig. 8) of Sapporo Maruyama Zoo on October 14, 2018. The workshop was by appointment only. Numbered tickets were given at noon on the day of the event; the hall had a capacity of 12 people per session. Each session consisted of a 40-min tour and was held four times in total. At the time of registration, we explained that the photos and the results of the questionnaires will be used for publicity and research and that personal information will be kept confidential.

\subsubsection{Participants}

In total, 39 elementary students (14 boys and 25 girls) mainly from the third to fifth grade participated in the event, and for $>70 \%$, our workshop was their first experience of a VR product, as shown in Fig. 9.

In the workshop, participants were limited to those older than the third grade in elementary school for two reasons. First, in accordance with the guidelines published by Location-based VR Association $\mathrm{Inc}^{10}$., VR videos are not recommended for children younger than seven years (first or second grade in elementary school in Japan) because they can harm the development of special perception. In addition, considering the difficulties of the concepts in the video contents, such as special frequency, CCF, and wavelength of light, we recommended children above the third grade, which is when they begin learning about science.

\subsubsection{Procedure}

The workshop took place in the form of a tour inside a fictional "research facility on animal vision." The participants were assigned to one group of four at the booking step. During the tour, parents waited for the participants in the waiting area, as shown in Fig. 8.

First, all 12 participants gathered in front of the LCD screen. Then, the fictional "Chief of the Institute" greeted the audience and gave them some tips regarding watching VR videos ("Please, keep your hands on the headset while watching the videos," "Do

\footnotetext{
${ }^{10}$ https://lva.or.jp/en/index.html
}

H. Kasuga et al.

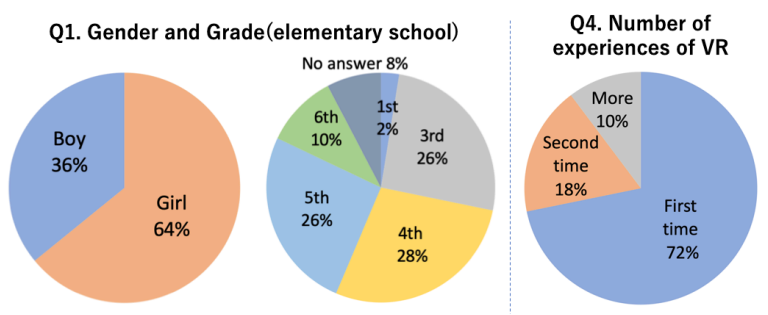

Figure 9: Participants' gender, grades, and number of experiences with VR content in the workshop in accordance with their answers to Q1 and Q4.

not get up from your chair while wearing the headset to avoid accidentally hitting the people around you." etc.) for approximately $3 \mathrm{~min}$. Then, we handed out a quiz sheet to ask the students to write a "report" summarizing the results of their studies while touring the institute. The guidance staff then went over the visual, color, and dynamic vision "labs" in order. In each "lab," the staff provided explanations using posters. One staff member was assigned to each pair of children to ensure that they were in no danger while watching the videos. While the participants were watching the VR videos, the staff asked "What do you see now? Are you feeling any VR motion sickness? Are you okay?" and supervised the participants.

After the explanation and the VR video, the participants wrote answers on the quiz sheet. To keep the VR headsets hygienic, the explanatory staff wiped the headsets with a sanitizing sheet during each group's turnover. The time spent in each "lab" was approximately $10 \mathrm{~min}$. After all the "lab" visits were complete, all groups gathered in front of the LCD screen. At the end of the visit, the "Laboratory Director" provided a few remarks. During his greeting, he stated in the VR video that "Numerous studies have focused on animal vision using various methods; however, what animal vision really looks like is not understood until we attempt to become them. Furthermore, there are individual differences even within the same species. I want you to think about why there is such diversity." After the tour, the children completed their questionnaires.

The contents of the quiz sheet were as follows. For the eyesight quiz, the participants placed stickers for each animal in the frame, starting from the left, in descending order of eyesight quality using clues about the ecology of turtles, hawks, and moles, as shown in Fig. 10. For the color vision quiz, two pictures of cat food with red coloring were provided that had been processed to either a normal color or a dichromatic tone. To determine the pictures that represented human and cat vision, the participants placed stickers on each cat food picture. For the kinetic acuity quiz, the children put stickers to represent each animal in the frame from left to right in descending order of kinetic acuity using clues regarding their body size and metabolism for squirrels, pigeons, and frogs. After the workshop, the children took the quiz sheet home as a souvenir.

\subsubsection{Questionnaire}

The items on the questionnaire were as follows:

Q1: What is your gender and grade in school?

Q2: How did you learn about this event? (Multiple answers allowed)

Q3: What motivated you to participate in this event? 

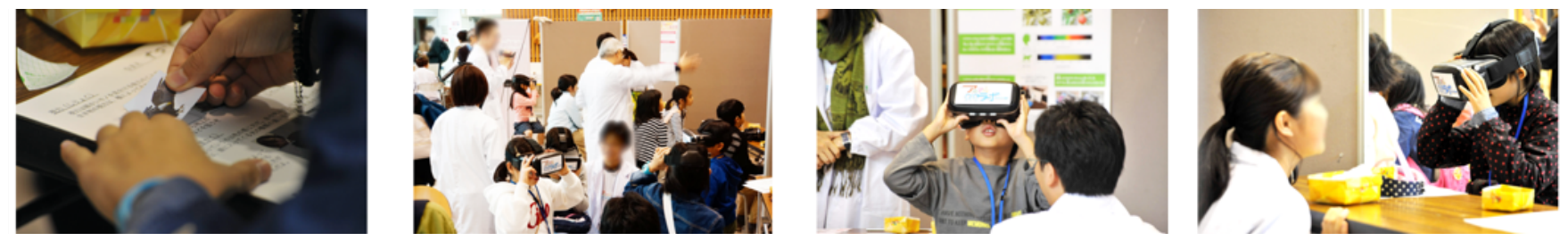

Figure 10: Stickers for the sheet.

Figure 11: Staff supporting children during the workshop.

(Multiple answers allowed)

Q4: Have you ever experienced any virtual reality content? (Single answer)

Q5: Which animals did you like in our VR video? (Multiple choice allowed)

Q6: Which kind of other animals would you want to see in virtual reality videos? (Open question)

Q7: Do you think the staff's explanation of how to use the VR headset was easy to understand? (Seven-point Likert question)

Q8: Do you think that the VR video on animals' eyesight was good for learning? (Seven-point Likert question)

Q9: Do you think that the VR video on animals' dynamic vision was good for learning? (Seven-point Likert question)

Q10: Do you think that the VR video on animals' color vision was good for learning? (Seven-point Likert question)

Q11: Would you want to participate in events to learn science using VR if you had the opportunity after our event? (Seven-point Likert question)

Q12: Are you inclined to want to use virtual reality products after our event? (Seven-point Likert question)

Q13: Do you want to learn more about animals' vision after our event? (Seven-point Likert question)

Q14: If you have any additional comments, like interesting points, about our event, please write them here. (Open question)

In the seven-point Likert questions, "7" denoted the most positive, whereas " 1 " denoted the most negative evaluation.

\subsubsection{Results of the questionnaire in the workshop}

The workshop was held safely, as per the measures shown in Fig. 11.

All 39 participants answered the questionnaire. Fig. 12 (Q2) indicates that almost half of the participants $(\mathrm{N}=18)$ obtained information regarding the workshop through our university website, whereas only one participant checked the website of either NoMaps or the zoo. Over a quarter of all participants just happened to visit the zoo and noticed the workshop. Most participants had pre-planned to participate in the workshop.

Regarding the companions who brought the participants to the workshop, the number of those who chose "by themselves $(\mathrm{N}=$ 21)" was over twice that of those who answered "by parents $(\mathrm{N}=$ 4)" or "by friends $(\mathrm{N}=6)$," as shown in Fig. $12(\mathrm{Q} 3)$. More than half of the participants came to the workshop with intrinsic rather than extrinsic motivations. Almost the same number of participants came to the workshop because of their interest in animal vision $(\mathrm{N}=18)$ and to experience VR products $(\mathrm{N}=19)$, as indicated in Fig. 12 (Q3).

Although the number of participants who answered that they liked cats $(\mathrm{N}=23)$ and dogs $(\mathrm{N}=20)$ was more than double the number of those who liked frogs $(\mathrm{N}=7)$ or geckos $(\mathrm{N}=8)$, as shown in Fig. $12(\mathrm{Q} 4)$, the participants answered that they were interested in the vision of various animal species in the open question, as shown in Fig. 13. In addition, two participants described why they wanted to see other animals in Q6: "Large animals like elephants, tigers." (P9: third-grade boy) and "Animals with very good eyesight like hawks/eagles" (P37: sixth-grade girl).

As shown in Fig. 14, most participants scored 6 or 7 in Q7-13.

In the open question for any additional comments (Q14), 36 of the 39 participants provided comments.

Out of the 36 participants, 29 not only commented on the experience, such as I had fun" (P6: third-grade girl) but also provided comments such as $I$ learned the color vision of animals!" (P36: sixth-grade girl) using words such as "vision," "saw," or "science." Twenty-two of these 29 participants mentioned something related to what the videos showed or what the staff explained; for example, "It was interesting that even among animals, the eyesight of animals differs. " (P37: sixth-grade girl) and 'At first, I thought that humans have the same color vision as all other animals. This is not the case, which surprised me." (P29: third-grade girl) No participants mentioned all three factors concretely.

Eleven participants used the term "virtual reality" or "VR"; for example, "it was easier to learn about them while experiencing it in virtual reality" (P23: fifth-grade boy).

Many participants mentioned "differences between vision of human and other animals;" for example "I learned that other animals have different vision from humans" (P22: third-grade boy), which led to further questions such as "It was interesting

Q2. How did you learn about this event?

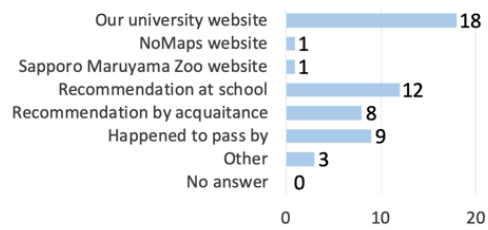

Q3. What motivated you to participate in this event?

$$
\begin{array}{rrr}
\text { My own motivation } & & 21 \\
\text { Recommendation by parents } & 4 & \\
\begin{array}{r}
\text { Recommendation by friend } \\
\text { Interest in animal's vision }
\end{array} & 6 & 18 \\
\text { Interest in the VR experience } & & 19 \\
\text { Other } & 1 & \\
\text { No answer } & 0 & \\
& 0 & 20
\end{array}
$$

Q5. Which animals did you like in our VR video?

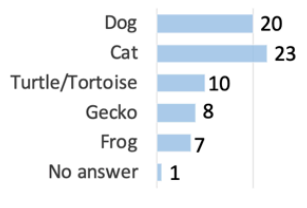

$0 \quad 20 \quad 40$

Figure 12: Results of Q2, Q3, and Q5 in the zoo workshop (multiple choices). 


\section{Q6. Which kind of other animals would you want to see in virtual reality video?}

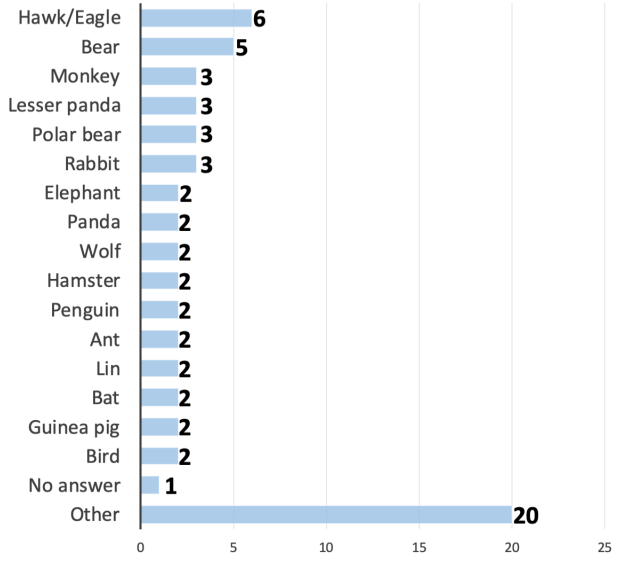

Figure 13: Result of Q6 in the zoo workshop (open question).

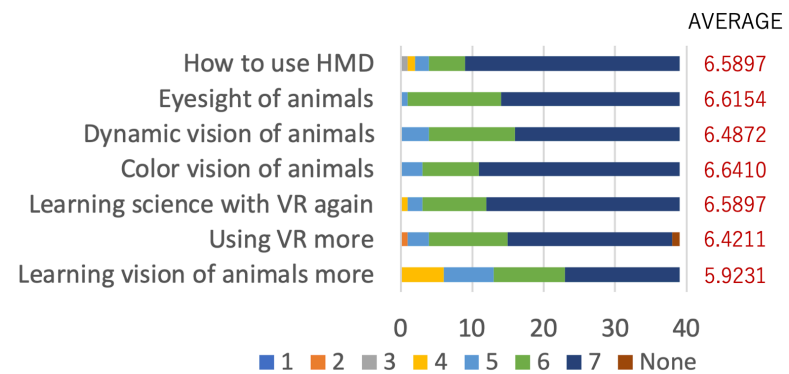

Figure 14: Results of Q7-13 in the zoo workshop (sevenpoint Likert question where " 7 " is the most positive and " 1 " is the most negative).

that the color vision of cats and dogs are different from ours. I am curious about whether cat-food looks delicious to cats" (P32: sixth-grade girl).

\subsection{Booth Exhibition at Equimonia}

On December 1-2, 2018, Equimonia 2018 was held at Kobe Sambo Hall in Japan. Approximately 1.5 times as many groups as the number of booths available applied to participate in Equimonia 2018. After the selection process, 200 booths, including ours, were allowed to exhibit. The size of each booth for the exhibition was $1.8 \mathrm{~m}$ wide, $1.2 \mathrm{~m}$ deep, and $2.1 \mathrm{~m}$ high.

\subsubsection{Participants}

The exact number of people who visited our booth was unknown because approximately 5,800 people freely came and went. In total, 196 people responded to the survey ( 98 on the first day and 98 on the second day; 69 men and 125 women; they belonged to various professions and age groups), as shown in Fig. 15. For approximately half of the participants, it was the first experience of a VR product. However, approximately $23 \%$ of them had experienced such technology more than twice before (Fig. 15).

\subsubsection{Procedure.}

Participants spoke to us while trying the VR videos. We wiped the headsets with disinfectant wipes and then explained some precautions such as "please do not walk around while you are
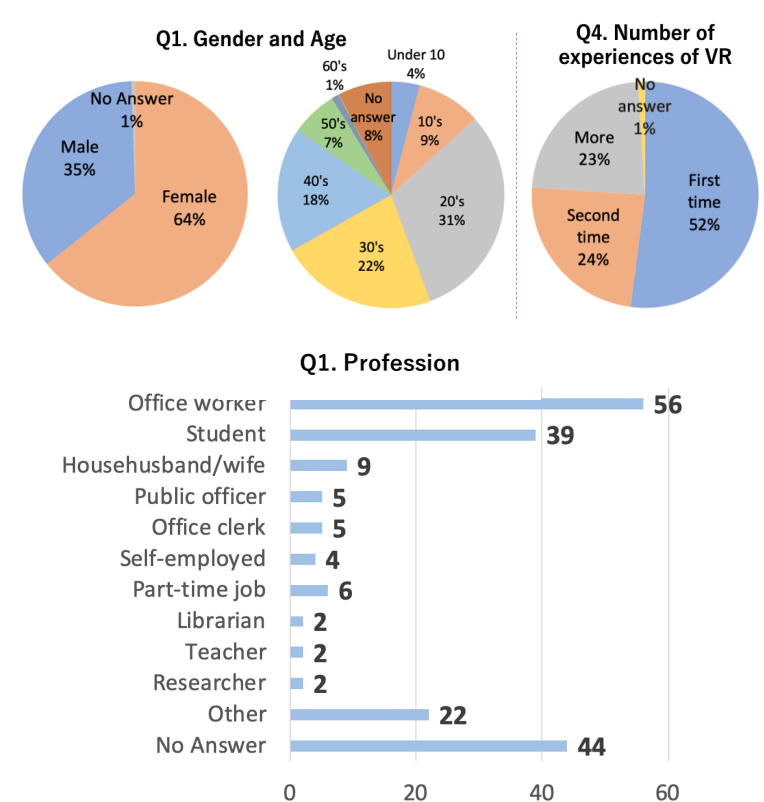

Figure 15: The gender, age, profession and times of their experiences with VR content of respondents in the booth exhibition in accordance with the answers to Q1 and Q4

wearing the headset to avoid accidentally hitting others" and "let us know if you feel dizzy. It sometimes happens to people watching VR videos." During and after the participants watched the videos, we chatted with them to answer any questions. After watching the video, we gave them a booklet explaining our VR video and asked them to fill in a questionnaire. The participants could freely read our explanation posters.

\subsubsection{Questionnaire}

We used almost the same questionnaire as that used at the zoo event. We modified Q1 from asking about school grades to asking about professions and Q2 from advertising at the zoo, school, or NoMaps website to advertising at the corporate website related to our project because the cooperating partners at Equimonia are different from those of our workshop at NoMaps, and we deleted the option "by parents" from Q3 because we assumed that most participants in Equimonia would be adults.

\subsubsection{Results of the questionnaire in the booth exhibition}

It was difficult for all participants to experience all three of our VR videos in the booth exhibition format as many individuals passed by and walked by our stall, as shown in Fig. 16 (Q2). Therefore, the results of the questionnaire, particularly the perception of eyesight effectiveness (Q8), dynamic vision (Q9), and color vision (Q10) experiences using VR videos in learning about animal ecology, were uncontrolled.

Seventy percent of respondents $(\mathrm{N}=136)$ attended our exhibition booth because they happened to walk nearby, as shown in Fig. 17 (Q2). Only two respondents visited our university's website. They had not planned to visit our booth beforehand.

The respondents visited our booth with intrinsic motivation $(\mathrm{N}=$ 57), as shown in Fig. 16 (Q3), which also showed that approximately half of the respondents visited our booth due to interest in animal vision $(\mathrm{N}=82)$, and over half of the respondents were motivated by their interest in experiencing VR 
Exploring the Needs and Ways to Use Virtual Reality to Understand Animals' Perceptions

\section{Q2. How did you learn about this event?}

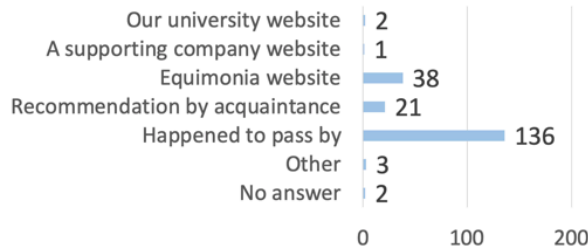

Q3. What motivated you to participate in this event?

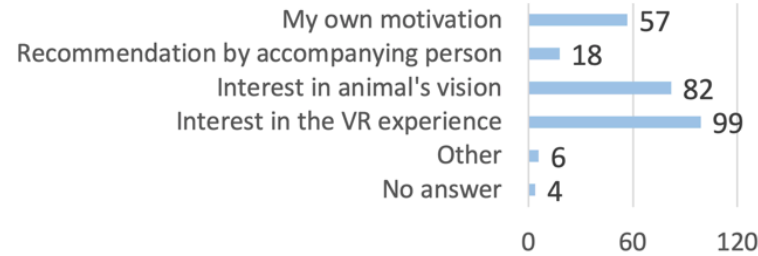

Q5. Which animals did you like in our VR video?

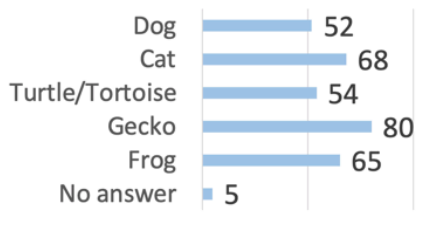

$0 \quad 50 \quad 100$

Figure 16: Results of $Q 2, Q 3$, and $Q 5$ in the booth exhibition (multiple choices)

products.

Fig. 16 (Q5) indicates that the votes for animals the respondents liked in our videos were not concentrated on cats and dogs; the votes were relatively split.

In the open question, although respondents were interested in the vision of various animal species, the vision of birds was the most popular, as shown in Fig. 17. Ten respondents described why they had chosen the animal; for example, respondents said "insects (compound eyes)" (P163: 49-year-old male office worker), "insects (ultraviolet rays)" (P135: 25-year-old female environmental researcher), and "animals that have a wider field of vision" (P197: 23-year-old female student in graduate school), and "world from the viewpoint of nocturnal animals" (P114: 23year-old male student).

As shown in Fig. 18, most participants scored 6 or 7 in Q7-Q13.

In the open question for any additional comments (Q14), 65 out of the 196 respondents wrote comments.

Nine respondents used the term "virtual reality" or "VR" in their comments. For example, "It was easier to understand the differences between the dichromacy of cats and dogs and the trichromacy of humans. I think it would be difficult to imagine how a gecko looks like using only words, so I am happy I was able to experience it through the VR." (P102: 18-year-old female student) This highlights the effectiveness of HMD-VR to help people grasp the focused characteristics of the vision of different animals. Unfortunately, two out of the nine and one other respondent stated that they experienced VR motion sickness.

Many respondents mentioned animal vision; for instance, participants responded "...especially, I have heard about the color vision of cats. However, it was difficult to understand that without such an experience" (P142: female office worker), "If they cannot

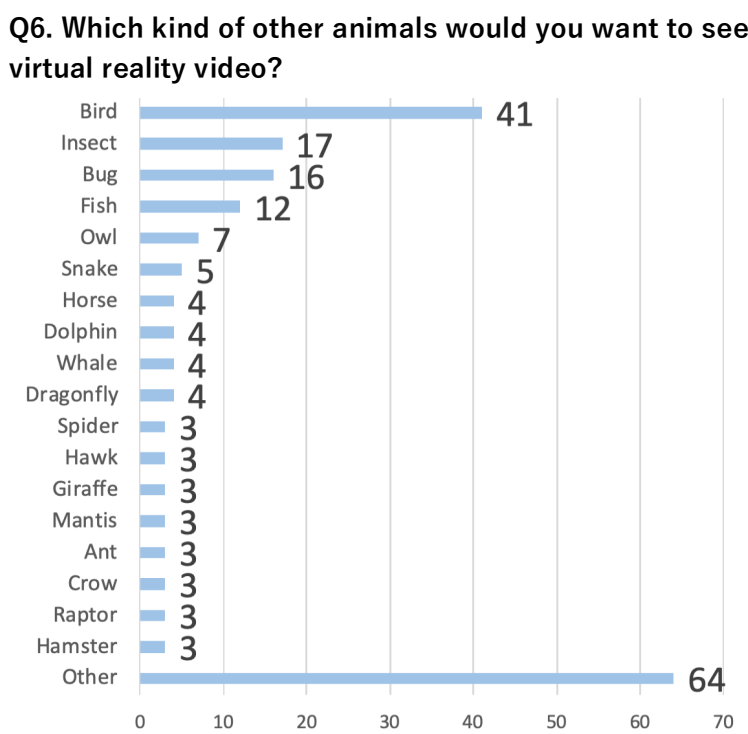

Figure 17: Results of Q6 at the booth exhibition (open question).

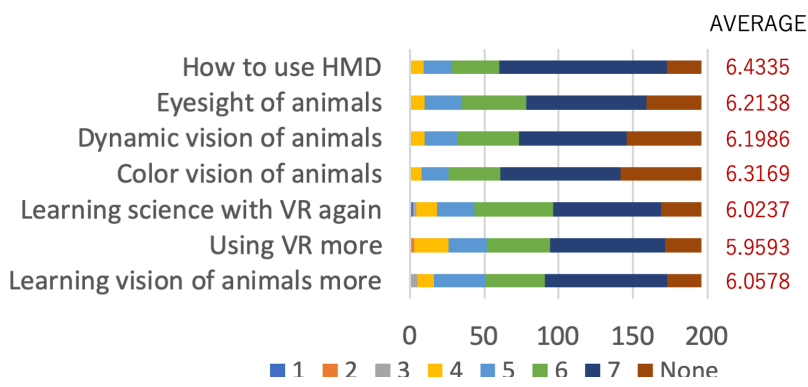

Figure 18: Results of Q7-13 in the booth exhibition (sevenpoint Likert question where " 7 " is the most positive and

" 1 " is the most negative).

distinguish colors, I wonder if the colors of toys are for humans" (P76: 55-year-old female housewife), "I would like to see the difference in vision between various predators..." (P115: 22-yearold female student), "Simulating animals' vision allows us to notice some points which we should understand while keeping companion animals" (P39: 30-year-old female part-time worker), and "before, I thought that cats and dogs could distinguish between black and white. To my surprise, I found that they could easily perceive the color green" (P11: 30-year-old male civil officer).

There were also respondents who provided the following advice: "It would be better if you could explain more about how animals see things (e.g., body structure)" (P44: 24-year-old female office worker) and "I want more explanations related to animals' ecology" (P113: male).

Interestingly, there were comments related to respondents' profession, such as "I wanted to produce a VR related to the human body's immune response" (P117: 35-year-old male researcher), "I think I want to try them as educational tools at school" (P112: 32-year-old male teacher), and "They were interesting because they were different from what I had ever imagined" (P194: 36-year-old male zookeeper). 
ACI2020, November 2020, Milton Keynes, United Kingdom

\section{DISCUSSION}

The results of our field studies at NoMaps and Equimonia imply the following:

- There were differences in the efficacy of advertisements, depending on the characteristics of the science event.

- There were differences in the animal species in which the participants were interested, depending on the characteristics of the science event.

- HMD-VR can allow people to understand the characteristics of animal vision more easily.

- Focusing on one characteristic of animal vision in a product can help people understand vision characteristics.

- Instructors may lead to a deeper and more correct understanding of animals.

First, half of the zoo workshop attendees, who were co-sponsored by NoMaps, came after visiting our university's website. At Equimonia, only two of the 196 survey respondents referred to the university's website; $70 \%$ of the questionnaire respondents visited our booth because they happened to walk past the booth. Although it is natural, the advertising strategy for science events differs depending on the event's format, such as exhibiting as a booth among hundreds or thousands of others or exhibiting as only one event at the place on that day.

Second, depending on the different demographics of the event attendees, the animal species that people wanted to view also changed. Among the VR videos viewed at NoMaps, the most popular animals were dogs and cats, which are the most common household companion animals. Meanwhile, the votes were split at Equimonia, with geckos being particularly popular. In addition, when asked about other animals that the participants would like to see in VR, many children at NoMaps stated the popular animals at the zoo. However, at Equimonia, some participants were interested in creatures with special vision, such as compound eyes, ultraviolet vision, wider field of vision, and nocturnal animal vision. This may be due to the difference in demographics: NoMaps targeted schoolchildren, whereas Equimonia mainly focused on adults who were animal fanatics and who went out of their way to attend an event for animal enthusiasts.

Third, according to the high scores in the Likert question section and examples mentioned in the free writing section, HMD-VR made understanding animal vision easier for attendees of both NoMaps and Equimonia. It is to be noted that high scores evaluated by users alone do not mean that the educational products are helpful for the user to learn something properly. Those who thought they learned information properly without misunderstandings could also evaluate the products with a high score. However, the descriptions in the free writing section provided examples that indicate that some participants understood the concepts of our videos properly. For instance, it may be difficult for elementary school-aged children to understand FPS or $\mathrm{CFF}$ even if it is explained to them beforehand in words. However, the HMD-VR experience provides opportunities to recognize that "when the FPS drops, the video appears to move frame-by-frame." Thus, an intuitive understanding of the perception of others through VR will lead to the application of ACI in providing a way to facilitate the understanding of animals. This can be useful in making people think about the welfare of the

animals we keep as pets. Therefore, it is important for any participant group to first achieve an intuitive understanding and interest through VR and then guide them to the correct understanding through explanations.

Regarding the characteristics of vision mentioned in the openended responses, participants almost exclusively mentioned only one of the three characteristics at most. Thus, it may be difficult to make a large impression on many visual characteristics together, particularly at a NoMaps event attended by elementary school students. If it is difficult to educate the participants regarding more than one visual feature at a time, even if the visual features are extracted and described, it is assumed that it will be more difficult to experience VR that implements more than one visual feature at a time.

There were a few references for instructors in open-ended questionnaire sections. However, as mentioned in previous studies [16], instructors may be necessary to communicate the limits of representation and the limitations of human perception in an exhibition on the subject of vision, which may be prone to misinterpretation. In addition, at Equimonia, the content was very easy for adults because it was aimed at children, and some of the participants wanted additional information such as body structure or ecology. At such times, the presence of an instructor may lead to a deeper understanding of the animal.

\section{CONCLUSION}

Research on the application of HMD-VR to education has exponentially increased in recent years. The advantages of HMDVR in education include immersive power and intuitive simplicity.

We produced VR videos to teach participants about the factors related to vision, including acuity, color vision, and dynamic vision, using videos filmed with a $360^{\circ}$ camera in a real environment where the animals were housed. Furthermore, we used a questionnaire survey to gauge the needs and usage of VR as a tool for improving human-animal relationships in two different fields: a workshop for 39 children at a zoo and an exhibition booth, where 196 attendees answered the questionnaire. Thus, although our videos did not provide a complete representation of animal vision, it was suggested that i) by isolating the elements and ii) by instruction and mentioning the limitations of VR, HMD-VR could be effectively used to promote awareness of the differences between humans and animals. The types of animals the participants wanted to see as subjects tended to differ between the workshop at the zoo and the booth exhibition in the Equimonia festival depending on the participant demographics. Differences in the knowledge of participants of animal life may have influenced this.

In the future, we would like to investigate the effectiveness of combining VR with non-VR content to produce an entire event.

We will make the best use of waiting time as it is difficult to have access to numerous VR devices and instructors at the same time. This is expected to enhance the immersive experience of VR.

In addition, we would like to evaluate the learning effect on people who have experienced VR multiple times. A high percentage of participants were mostly motivated by VR itself as a primary factor and had not used VR before. Therefore, high scores in the Likert question section may be due to the novelty associated with VR. 
Exploring the Needs and Ways to Use Virtual Reality to Understand Animals' Perceptions
ACI2020, November 2020, Milton Keynes, United Kingdom

\section{REFERENCES}

[1] Jakob von Uexkull and Georg Kriszat. 1934. Streifzuge durch die Umwelten von Tieren und Menschen: Ein Bilderbuch unsichtbarer Wellen. Springer Berlin Heidelberg, (Jan. 1934). DOI: https://doi.org/10.1007/978-3-642-98976-6

[2] Gordon M Burghardt, 1990. Animal suffering, critical anthropomorphism, and reproductive rights. Behavioral and Brain Sciences 13, 1 (1990), 14-15. DOI: https://dx.doi.org/10.1017/S0140525X00077153

[3] Clara Mancini, 2011. Animal-computer Interaction: A Manifesto. Interactions 18, 4 (Jul. 2011), 69-73. DOI: http://dx.doi.org/10.1145/1978822.1978836

[4] Clint Zeagler, Jay Zuerndorfer, Andrea Lau, Larry Freil, Scott Gilliland, Thad Starner, and Melody Moore Jackson. 2016. Canine Computer Interaction: Towards Designing a Touchscreen Interface for Working Dogs. In Proceedings of the Third International Conference on Animal-Computer Interaction ( $\mathrm{ACl}$ '16). ACM Press, New York, NY, USA, Article 2, 5. DOI: http://dx.doi.org/10.1145/2995257.2995384

[5] Clint Zeagler, Scott Gilliland, Larry Freil, Thad Starner, and Melody Jackson. 2014. Going to the Dogs: Towards an Interactive Touchscreen Interface for Working Dogs. In Proceedings of the 27th Annual ACM Symposium on User Interface Software and Technology (UIST '14). ACM Press, New York, NY, USA, 497-507. DOI: http://dx.doi.org/10.1145/2642918.2647364

[6] Clara Mancini, Rob Harris, Brendan Aengenheister, and Claire Guest. 2015. ReCentering Multispecies Practices: A Canine Interface for Cancer Detection Dogs. In Proceedings of the 33rd Annual ACM Conference on Human Factors in Computing Systems (CHI '15). ACM Press, New York, NY, USA, 2673-2682. DOI: http://dx.doi.org/10.1145/2702123.2702562

[7] Melody Moore Jackson, Clint Zeagler, Giancarlo Valentin, Alex Martin, Vincent Martin, Adil Delawalla, Wendy Blount, Sarah Eiring, Ryan Hollis, Yash Kshirsagar, and Thad Starner. 2013. FIDO - facilitating interactions for dogs with occupations: wearable dog-activated interfaces. In Proceedings of the 2013 International Symposium on Wearable Computers (ISWC '13). Association for Computing Machinery, New York, NY, USA, 81-88. DOI: https://doi.org/10.1145/2493988.2494334

[8] Sarah Webber, Marcus Carter, Sally Sherwen, Wally Smith, Zaher Joukhadar, and Frank Vetere. 2017. Kinecting with Orangutans: Zoo Visitors' Empathetic Responses to Animals? Use of Interactive Technology. In Proceedings of the $2017 \mathrm{CH}$ Conference on Human Factors in Computing Systems (CHI '17). ACM Press, New York, NY, USA, 6075-6088. DOI: http://dx.doi.org/10.1145/3025453.3025729

[9] John Fass and Kevin Walker. 2016. De-computing the pigeon sensorium. In Proceedings of the Third International Conference on Animal-Computer Interaction (ACl '16). Association for Computing Machinery, New York, NY, USA, Article 12, 1-4. DOI:https://doi-org.ezoris.lib.hokudai.ac.jp/10.1145/2995257.3012022

[10] Dejian Liu, Kaushal Kumar Bhagat, Yuan Gao, Ting-Wen Chang, and Ronghuai Huang. 2017. The Potentials and Trends of Virtual Reality in Education. Virtual, Augmented, and Mixed Realities in Education. Smart Computing and Intelligence. Springer, Singapore. DOI: https://doi.org/10.1007/978-981-10-5490-7_7

[11] Sally Lindsay and Ashley Edwards, 2013. A systematic review of disability awareness interventions for children and youth. Disability and Rehabilitation 35, 623-646. DOI: https://doi.org/10.3109/09638288.2012.702850

[12] Jani Väyrynen, Ashley Colley, and Jonna Häkkilä. 2016. Head mounted display design tool for simulating visual disabilities. In Proceedings of the 15th Internationa Conference on Mobile and Ubiquitous Multimedia (MUM '16). Association for Computing Machinery, New York, NY, USA, 69-73. DOI: https://doi.org/10.1145/3012709.3012714

[13] Jun Nishida, Soichiro Matsuda, Mika Oki, Hikaru Takatori, Kosuke Sato, and Kenji Suzuki. 2019. Egocentric Smaller-person Experience through a Change in Visual Perspective. In Proceedings of the 2019 CHI Conference on Human Factors in Computing Systems (CHI '19). Association for Computing Machinery, New York, NY, USA, Paper 696, 1-12. DOI: https://doi.org/10.1145/3290605.3300926

[14] Maria Roussou. 2001. Immersive Interactive Virtual Reality in the Museum. In Proceedings of TiLE - Trends in Leisure Entertainment. London, UK: Aldrich, UK.

[15] Daniel A. Guttentag, 2010. Virtual reality: Applications and implications for tourism, Tourism Management 31, 5, 2010, 637-651. DOI: https://doi.org/10.1016/j.tourman.2009.07.003.

[16] Göran Fransson, Jörgen Holmberg, and Claes Westelius, 2020. The challenges of using head mounted virtual reality in K-12 schools from a teacher perspective. Education and Information Technologies, 1-22. DOI: https://doi.org/10.1007/s10639-020-10119-1

[17] Randolph L. Jackson and Eileen Fagan. 2000. Collaboration and learning within immersive virtual reality. In Proceedings of the third international conference on Collaborative virtual environments (CVE'OO). Association for Computing Machinery, New York, NY, USA, 83-92. DOI: https://doi.org/10.1145/351006.351018

[18] Rula Al-Azawi, Ali Albadi, Raziyeh Moghaddas, and Jonathan Westlake. 2019.
Exploring the Potential of Using Augmented Reality and Virtual Reality for STEM Education: Learning Technology for Education Challenges. LTEC 2019. Communications in Computer and Information Science, 1011. Springer, Cham. DOI: https://doi.org/10.1007/978-3-030-20798-4_4

[19] Diego Iquira, Briseida Sotelo, and Olha Sharhorodska. 2019. A Gamified MobileBased Virtual Reality Laboratory for Physics Education: Results of a Mixed Approach. $\mathrm{HCl}$ International 2019 - Posters. HCll 2019. Communications in Computer and Information Science, 1034. Springer, Cham. DOI: https://doi.org/10.1007/978-3-03023525-3_32

[20] Rogers Christian B., El-Mounayri Hazim, Wasfy Tamer, and Satterwhite Jesse, 2018. Assessment of STEM e-Learning in an Immersive Virtual Reality (VR) Environment. The ASEE Computers in Education (COED) Journal 8, 4.

[21] Tomas Blazauskas, Rytis Maskeliunas, Reda Bartkute, Vitalija Kersiene, Indre Jurkeviciute, and Mindaugas Dubosas. 2017. Virtual Reality in Education: New Ways to Learn. Information and Software Technologies. ICIST 2017. Communications in Computer and Information Science, 756. Springer, Cham. DOI: https://doi.org/10.1007/978-3-319-67642-5_38

[22] Adeola Fabola and Alan Miller. 2016. Virtual Reality for Early Education: A Study. Immersive Learning Research Network. iLRN 2016. Communications in Computer and Information Science, 621. Springer, Cham. DOI: https://doi.org/10.1007/978-3 319-41769-1_5

[23] Don Allison, Brian Wills, Doug A. Bowman, Jean D. Wineman, and Larry F. Hodges. 1997. The Virtual Reality Gorilla Exhibit in IEEE Computer Graphics and Applications 17, 6 (Nov. 1997), 30-38. DOI: https://doi.org/10.1109/38.626967.

[24] Doug Bowman, Larry Hodges, Don Allison, and Jean Wineman. 2001. The Educational Value of an Information-Rich Virtual Environment. Presence: Teleoperators and Virtual Environments. 8. DOI: https://doi.org/10.1162/105474699566251

[25] Randolph Blake, Steven J. Cool, and M.L.J. Crawford. 1974. Visual resolution in the cat. Vision Research 14, 11, 1211-1217. DOI: https://doi.org/10.1016/00426989(74)90218-1

[26] Eleanor M Caves, Nicholas C Brandley, and Sönke Johnsen. 2018. Visual Acuity and the Evolution of Signals. Trends in Ecology \& Evolution 33, 5 358-372. DOI: https://doi.org/10.1016/j.tree.2018.03.001

[27] Robert Fox, Stephen W. Lehmkuhle, and David H. Westendorf. 1976. Falcon visual acuity. Science 192, 4236, 263-265. DOI: https://doi.org/10.1126/science.1257767

[28] Northmore, D.P. and Granda, A.M. 1991. Refractive state, contrast sensitivity, and resolution in the freshwater chelonian, Pseudemys scripta elegans, determined by tectal visual-evoked potentials. Visual Neuroscience 7, 6, 619-625. DOI: https://doi.org/10.1017/s0952523800010403

[29] Robert A. Wilson and Frank Keil. 1999. The MIT Encyclopedia of the Cognitive Sciences (MITECS). MIT Press, 1999, 147-148.

[30] Marcello Siniscalchi, Serenella d'Ingeo, Serena Fornelli, and Angelo Quaranta. 2017. Are dogs red-green colour blind?. Royal Society Open Science 4, 170869. DOI: https://doi.org/10.1098/rsos.170869.

[31] N. W. Daw and A. L. Pearlman. 1970. Cat colour vision: evidence for more than one cone process. J Physiol 211, 1 (1970), 125-137. DOI: https://doi.org/10.1113/jphysiol.1970.sp009270

[32] Clint Zeagler, Scott Gilliland, Larry Freil, Thad Starner, and Melody Jackson. 2014. Going to the dogs: Towards an interactive touchscreen interface for working dogs. UIST 2014 - Proceedings of the 27th Annual ACM Symposium on User Interface Software and Technology. 497-508. DOI: https://doi.org/10.1145/2642918.2647364.

[33] Françoise Viénot, Hans Brettel, and John D. Mollon. 1999. Digital video colourmaps for checking the legibility of displays by dichromats. Color Research \& Application 24, 4, 243-252. DOI: https://doi.org/10.1002/(SICI)15206378(199908)24:4<243::AID-COL5>3.0.CO;2-3

[34] Kevin Healy, Luke McNally, Graeme D. Ruxton, Natalie Cooper, and Andrew L. Jackson. 2013. Metabolic rate and body size are linked with perception of tempora information. Animal Behaviour 86, 4, 685-696. DOI: https://doi.org/10.1016/j.anbehav.2013.06.018

[35] Humberto R. Maturana, J. Y. Lettvin, W. S. McCulloch, and W. H. Pitts. 1960 Anatomy and physiology of vision in the frog (Rana pipiens). The Journal of general physiology 43, 6, 129-175. DOI: https://doi.org/10.1085/jgp.43.6.129

[36] Zoran Zivkovic. 2004. Improved adaptive Gaussian mixture model for background subtraction. In Proceedings of the 17th International Conference on Pattern Recognition, 2, 28-31. DOI: https://doi.org/10.1109/ICPR.2004.1333992. [37] Zoran Zivkovic and Ferdinand van der Heijden. 2006. Efficient adaptive density estimation per image pixel for the task of background subtraction. Pattern Recognition Letters 27, 7, 773-780. https://doi.org/10.1016/j.patrec.2005.11.005 